\title{
Plane-Wave Implementation and Performance of à-la-Carte Coulomb-Attenuated Exchange-Correlation Functionals for Predicting Optical Excitation Energies in Some Notorious Cases
}

\author{
Martin P. Bircher ${ }^{\circledR}$ and Ursula Rothlisberger*๑ \\ Laboratoire de Chimie et Biochimie Computationnelles, Ecole Polytechnique Fédérale de Lausanne, Lausanne 1015, Switzerland
}

Supporting Information

ABSTRACT: Linear-response time-dependent density functional theory (LR-TD-DFT) has become a valuable tool in the calculation of excited states of molecules of various sizes. However, standard generalized-gradient approximation and hybrid exchangecorrelation (xc) functionals often fail to correctly predict chargetransfer (CT) excitations with low orbital overlap, thus limiting the scope of the method. The Coulomb-attenuation method (CAM) in the form of the CAM-B3LYP functional has been shown to reliably remedy this problem in many CT systems, making accurate predictions possible. However, in spite of a rather con-

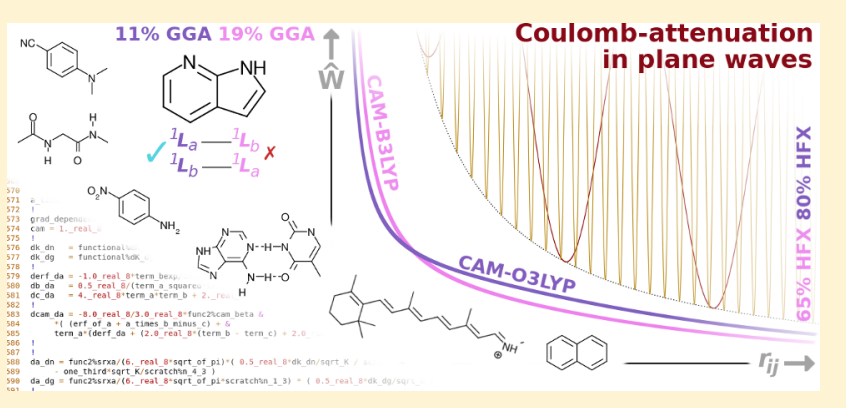
sistent performance across different orbital overlap regimes, some pitfalls remain. Here, we present a fully flexible and adaptable implementation of the CAM for $\Gamma$-point calculations within the plane-wave pseudopotential molecular dynamics package CPMD and explore how customized xc functionals can improve the optical spectra of some notorious cases. We find that results obtained using plane waves agree well with those from all-electron calculations employing atom-centered bases, and that it is possible to construct a new Coulomb-attenuated xc functional based on simple considerations. We show that such a functional is able to outperform CAM-B3LYP in some cases, while retaining similar accuracy in systems where CAM-B3LYP performs well.

\section{INTRODUCTION}

Be it for the vital conversion of sunlight to chemical energy in a leaf, for photochemical reactions causing harmful DNA damage to skin, for the blue fluorescence of scorpions, or for energy conversion in man-made solar cells: electronically excited states are of crucial importance to fundamental processes in Nature, and in scientific fields ranging from biology over chemistry to solid-state physics. The theoretical description of the excitations which are at the base of these phenomena makes it possible to ultimately gain an improved understanding of these key events.

A fully correlated description at the wave function level is, unfortunately, prohibitively expensive for many, if not most systems of relevant size. It is thanks to the considerable progress in the field of Kohn-Sham time-dependent density functional theory (KS-TD-DFT) $)^{1-3}$ that such excited-state processes can these days be described at a comparably moderate computational cost. The linear-response (LR) formulation of TD-DFT ${ }^{4}$ is routinely used by many a computational chemist, and the ever increasing availability of computational resources has made it possible to describe larger and larger systems. However, like ground-state DFT and even more so, the choice of an appropriate exchange-correlation (xc) functional, or respectively $\mathrm{xc}$ kernel, is crucial, and often decides between results in good agreement with high-level reference data, and spectra which are considerably red-shifted and exhibit an incorrect ordering of states.

The accuracy of the calculated transitions is mainly hampered by an inaccurate long-range decay of the xc potential, which leads to larger errors in the prediction of the excitation energies. ${ }^{5}$ Hence, in analogy to their tendency to underestimate HOMO-LUMO gaps, generalized-gradient approximation (GGA) ${ }^{6}$ xc functionals typically shift excitation energies by a considerable factor and may fail to recover the correct ordering of states. ${ }^{7}$ Hybrid functionals, ${ }^{8}$ which include a fixed proportion of exact exchange, may partly alleviate this problem for states in which there is an overlap between the orbitals involved in the transitions. However, even hybrid functionals will inevitably fail to describe low-overlap charge-transfer (CT) and Rydberg states, where the $1 / r$ decay of the Coulomb operator is an important constituent in the correct description of the interaction between spatially distant orbitals. ${ }^{9}$ But it is the inclusion of exact exchange that is most vital for these transitions: While an asymptotic correction of the (GGA) xc potential alone recovers the proper $1 / r$ dependency and improves the description of Rydberg states, it cannot successfully capture the effects of pronounced charge separation. ${ }^{7}$ A promising remedy to this problem has been found in an appropriate splitting of the Coulomb operator, making it possible to ensure a correct decay of the xc potential at long range, while keeping the accuracy and simplicity of a standard local formulation for the short-range components. The longrange correction (LC) scheme $\mathrm{e}^{5,10}$ and its generalization, the Coulomb-attenuation method (CAM), ${ }^{11}$ separate the Coulomb

Received: January 23, 2018

Published: April 16, 2018 
operator using an error function. While the short-range components are described using the GGA, the long-range components are taken into account using the exact exchange operator. This splitting captures the essentials of CT transitions and considerably increases the accuracy for such states. LC- and CAM-based $\mathrm{xc}$ functionals have therefore become a standard tool for the calculation of molecular excitation energies with pronounced CT character within TD-DFT. ${ }^{12}$ The correspondingly modified Coulomb operator is easily implemented in a Gaussian basis.

CAM-B3LYP is not only the first functional that was constructed using the CAM, it also has become probably the most prominent and abundantly used functional of this family. Peach et al. ${ }^{9}$ have assessed its performance on a diverse test set of maingroup molecules shortly after the original paper presenting CAM-B3LYP, and many successful applications of the functional followed: Among the substantial improvements documented with respect to $\mathrm{B} 3 \mathrm{LYP}^{8,13}$ or $\mathrm{PBE},{ }^{14}$ Peach et al. have found that CAM-B3LYP accurately describes excitations in (poly)acenes including naphthalene, yielding correct state ordering. They could also show that the spectra of model dipeptides improve substantially with respect to non-Coulomb attenuated functionals. ${ }^{7}$ Subsequently, other studies have found that the use of CAM-B3LYP can predict spectra of AT nucleobase dimers ${ }^{15}$ and indoles ${ }^{16}$ of varying substitution patterns with increased accuracy. All these systems exhibit CT or Rydberg transitions between spatially separated states, which results in the inevitable failure of GGA and conventional hybrid functionals.

The orbital overlap in a transition can be quantitatively characterized by the parameter $\Lambda$ introduced by Peach et al.; ${ }^{9}$ a small value of $\Lambda$ indicating a small overlap between the involved orbitals, a large value a substantial one. In their study covering excitations of different character, they have found that both GGA and conventional hybrids suffer from an inconsistent performance over the complete range of $\Lambda$ values: For overlap values $\Lambda<0.4$ in the case of PBE and $\Lambda<0.3$ in the case of B3LYP, the errors in the excitation energies become substantially bigger. For CAM-B3LYP, no such correlation was found over the whole range $0<\Lambda<1$. CAM-B3LYP was shown to fare particularly well for CT excitations, especially in the "low overlap" regime, $\Lambda<0.3$, although some cases with CT character in the "intermediate $\Lambda$ regime" are accurately described, too (e.g., the retinal protonated Schiff base $\left.{ }^{17,18}\right)$. However, in systems where there is significant overlap between the orbitals involved in the transition, conventional hybrid functionals such as PBE0 ${ }^{19,20}$ often fare better, and CAM-B3LYP tends to red-shift the excitation energies. This is notably the case for the doubly fluorescent dye DMABN, for which CAM-B3LYP tends to overestimate the excitation energies of the $S_{2}$ CT state with $\Lambda=0.72 .{ }^{9}$ Fully long-range corrected functionals ${ }^{21}$ such as LC-BLYP $^{5}$ or LC-PBE0 ${ }^{22}$ were not evaluated in the study by Peach et al., but they have since been shown to perform well in certain systems with very low overlap that cannot be accurately described with CAM-B3LYP. ${ }^{15,16,23}$ This is attributed to the absence of any GGA exchange at longest range, which benefits the description of Rydberg states, ${ }^{9,2}$ but comes at the cost of an inferior average performance especially for local excitations. ${ }^{11,16,21}$

Despite the absence of correlation between the predicted excitation energies and their $\Lambda$-values, there exist some systems even in the low-overlap range $(\Lambda<0.3)$ for which CAMB3LYP fails to deliver an accurate description. In the case of $p$-nitroaniline, the excitation energies are reasonably predicted, but solvatochromic shifts cannot be reproduced since the groundand excited-state dipoles are grossly over- and underestimated, respectively. ${ }^{24}$ Whereas CAM-B3LYP predicts a correct state ordering and reasonable energetics for the excitations in the AT base pair, ${ }^{15}$ the HOMO is predicted to lie on the wrong base when compared to higher-level wave function methods and basic considerations based on the ionization potential of the isolated bases. ${ }^{25}$ Seemingly reasonable results may therefore be obtained based on the wrong physical reason. Similarly, the ordering of close-lying, low-energy excitations may be inverted in some systems; this is the case for the $\beta$-dipeptide model system introduced by Serrano-Andrés et al. ${ }^{26}$ and subsequently popularized in the aforementioned benchmark set by Peach et al. ${ }^{9}$ Other studies have found the same problem to occur in the case of 7 -azaindole, ${ }^{16}$ even though the state ordering for other substituted indoles could be correctly predicted. A wrong ordering of states may be especially detrimental for excitedstate molecular dynamics, ${ }^{27}$ where the forces exerted on the nuclei may substantially differ between the two swapped states, leading to a quantitatively as well as qualitatively wrong propagation of the system. For systems such as the $\beta$-dipeptide and 7 -azaindole, ${ }^{16}$ the use of an LC functional may yield a qualitatively correct ordering of the low-lying excitations, but the energies often remain too low. Alternatively, the range separation parameter $\mu$ in CAM-B3LYP may be tuned in order to ameliorate the performance of the functional. ${ }^{28}$ This process known as $\gamma$-tuning adjusts the range separation parameter to a value that accurately reproduces ionization potentials. This approach often permits for an accurate description of the excitations of interest, ${ }^{16}$ but it constitutes a rather system-specific remedy, lacking portability and thus predictive power in comparing various systems. All of these notorious systems are included in Figure 1 and have been chosen here to test the performance of an "à-la-carte" combination of range-separated functionals.

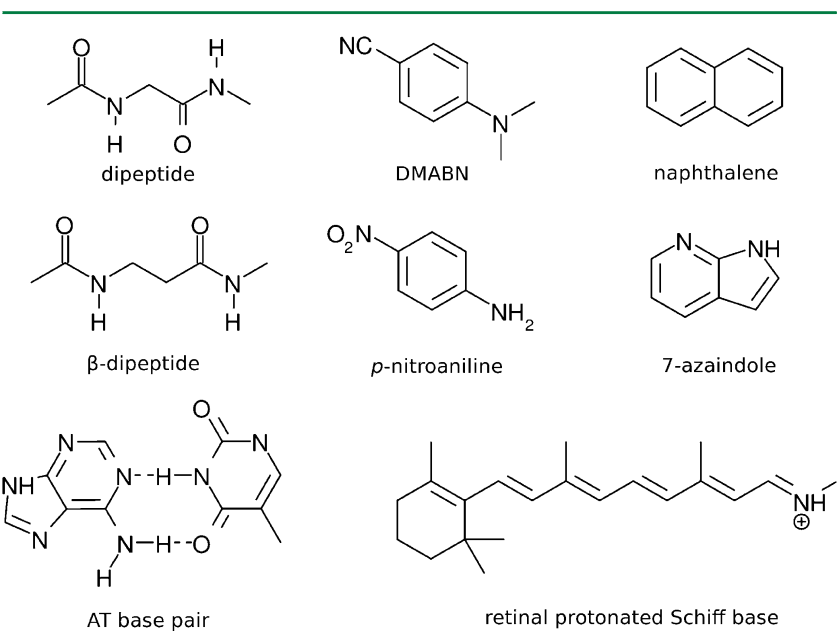

Figure 1. Molecules explicitly discussed in this study, all of which contain excitations that are difficult to describe in TD-DFT when using a GGA or hybrid functional as the xc kernel.

In other applications, a splitting of the Coulomb operator opposite to the LC and CAM scheme may be beneficial. This has been proposed in screened hybrid functionals for solid-state applications, ${ }^{29}$ where the exact exchange is limited to short range and the GGA exchange to long range. Functionals such as HSE $03^{29}$ yield results superior to those obtained with the GGA for many systems. Screened hybrids are especially beneficial in combination with a plane wave/pseudopotential approach, since they conveniently eliminate the divergence of the Coulomb operator at the $\mathbf{G}=0$ component of the plane wave basis in 
sufficiently large simulation cells. Since the splitting adopted in the LC and CAM schemes does not offer any particular advantage in solid-state applications and does not eliminate this problematic divergence term, these methods have received much less attention in plane wave codes.

In the following, we present the CAM applied to $\Gamma$-point calculations in a plane-wave pseudopotential framework. The implementation allows for the CAM to be combined with any exchange functional of choice, offering maximum flexibility. This makes the construction of customized "à-la-carte" Coulombattenuated xc functionals possible, which can be tailored to any system of choice, thus maximizing the performance of the method. In order to gain maximum flexibility, all CAM parameters can be chosen freely. Our implementation of the CAM in the molecular dynamics package CPMD ${ }^{30}$ targets applications where $\Gamma$-point sampling is routinely used, and makes the sampling of large systems possible via the fully Hamiltonian $\mathrm{QM} / \mathrm{MM}$ scheme implemented in the CPMD code. Simulations of CT systems in the gas phase as well as in condensed matter therefore become feasible using a plane wave/pseudopotential approach. To facilitate the calculation of the necessary terms, a new driver for the calculation of the xc energy has been implemented in the CPMD code.

The paper is organized as follows: First, we give a short summary of the CAM, followed by a description of the implementation. We then give a more detailed account of the test systems used to benchmark both the implementation and a new Coulomb-attenuated $\mathrm{xc}$ functional. We discuss the performance of the CAM in plane waves with respect to the choice of pseudopotential, and in comparison to all-electron calculations with atom-centered basis sets. Finally, we will show how a flexible choice of the underlying GGA exchange-functional can improve accuracy in systems where standard functionals yield unsatisfactory results by comparing a customized "à-la-carte" CAM-xc functional constructed based on simple considerations to the well-established CAM-B3LYP.

\section{THEORY}

2.1. The Coulomb-Attenuation Method (CAM). In the $\mathrm{CAM}$, the Coulomb operator $\hat{\mathrm{W}}$ is split into two domains dominated by long-range (lr) and short-range (sr) components each:

$$
\begin{aligned}
& \hat{W}=\hat{W}^{\text {sr }}+\hat{W}^{\mathrm{lr}} \\
& \left\langle\mathbf{r}^{\prime}\left|\hat{W}^{\mathrm{sr}}\right| \mathbf{r}\right\rangle=\frac{1-\left[\alpha+\beta \operatorname{erf}\left(\mu\left|\mathbf{r}-\mathbf{r}^{\prime}\right|\right)\right]}{\left|\mathbf{r}-\mathbf{r}^{\prime}\right|} \\
& \left\langle\mathbf{r}^{\prime}\left|\hat{W}^{\mathrm{lr}}\right| \mathbf{r}\right\rangle=\frac{\alpha+\beta \operatorname{erf}\left(\mu\left|\mathbf{r}-\mathbf{r}^{\prime}\right|\right)}{\left|\mathbf{r}-\mathbf{r}^{\prime}\right|}
\end{aligned}
$$

where $\alpha, \beta$, and $\mu$ are adjustable parameters ${ }^{11}$ and $\alpha=0$ and $\beta=1$ in the original LC method. ${ }^{5,10}$ The first term is treated using a GGA expression for the exchange functional and becomes smaller for larger Coulomb distances, whereas the second term grows with increasing $\left|\mathbf{r}-\mathbf{r}^{\prime}\right|$ and is treated using Fock's expression for the exchange energy.

The effective Coulomb operator in the exchange integrals then becomes

$$
\begin{aligned}
& E_{x}^{\mathrm{HFX}}=\frac{1}{2} \sum_{\sigma} \sum_{i} \sum_{j} \iint \psi_{i \sigma}^{*}(\mathbf{r}) \psi_{j \sigma}^{*}\left(\mathbf{r}^{\prime}\right) \times \\
& \frac{\alpha+\beta \operatorname{erf}\left(\mu\left|\mathbf{r}-\mathbf{r}^{\prime}\right|\right)}{\left|\mathbf{r}-\mathbf{r}^{\prime}\right|} \psi_{j \sigma}(\mathbf{r}) \psi_{i \sigma}\left(\mathbf{r}^{\prime}\right) \mathrm{d} \mathbf{r} \mathrm{d} \mathbf{r}^{\prime}
\end{aligned}
$$

Correspondingly, the GGA enhancement factor has to be adapted to the screened Coulomb operator. The adaptation is based on the LDA for a short-range Coulomb operator and appropriately generalized: ${ }^{5}$

$$
\begin{gathered}
E_{x}^{\mathrm{GGA}}=\frac{1}{2} \sum_{\sigma} \rho_{\sigma}^{4 / 3} K_{\sigma}\left\{1-\alpha-\beta \frac{8}{3} a_{\sigma} \times\right. \\
\left.\left[\sqrt{\pi} \operatorname{erf}\left(\frac{1}{2 a_{\sigma}}\right)+2 a_{\sigma}\left(b_{\sigma}-c_{\sigma}\right)\right]\right\}
\end{gathered}
$$

where $K_{\sigma}$ is the spin-dependent formulation of the exchange enhancement factor, and the terms due to the correction read

$$
\begin{aligned}
& a_{\sigma}=\frac{\mu K_{\sigma}^{1 / 2}}{6 \sqrt{\pi} \rho_{\sigma}^{1 / 3}} \\
& b_{\sigma}=\exp \left(-\frac{1}{4 a_{\sigma}^{2}}\right)-1 \\
& c_{\sigma}=2 a_{\sigma}^{2} b_{\sigma}+\frac{1}{2}
\end{aligned}
$$

2.2. Singularity of the Coulomb Operator $\hat{W}$ in Reciprocal Space. At the $\Gamma$-point, the exact exchange energy can be efficiently computed with a mixed real-space reciprocalspace algorithm ${ }^{31}$ after introducing a resolution of identity in $\mathbf{G}$ and rearranging the terms due to the complex conjugate of the Kohn-Sham (KS) orbitals (where $\left\langle\mathbf{r} \mid i_{\sigma}\right\rangle=\left\langle i_{\sigma} \mid \mathbf{r}\right\rangle$ at the $\Gamma$-point):

$$
\begin{aligned}
& E_{x}^{\mathrm{HFX}}=\sum_{\sigma} \sum_{i} \sum_{j \geq i} \int \mathrm{d} \mathbf{r}^{\prime}\left\langle j_{\sigma} \mid \mathbf{r}^{\prime}\right\rangle\left\langle\mathbf{r}^{\prime} \mid i_{\sigma}\right\rangle \times \\
& \int \mathrm{d} \mathbf{r}\left\langle\mathbf{r}^{\prime}|\hat{\mathrm{W}}| \mathbf{r}\right\rangle\left\langle i_{\sigma} \mid \mathbf{r}\right\rangle\left\langle\mathbf{r} \mid j_{\sigma}\right\rangle \\
& =\sum_{\sigma} \sum_{i} \sum_{j \geq i} \int \mathrm{d} \mathbf{r}^{\prime}\left\langle j_{\sigma} \mid \mathbf{r}^{\prime}\right\rangle\left\langle\mathbf{r}^{\prime} \mid i_{\sigma}\right\rangle \int \mathrm{d} \mathbf{G}^{\prime}\left\langle\mathbf{r}^{\prime} \mid \mathbf{G}^{\prime}\right\rangle \times \\
& \int \mathrm{d} \mathbf{G}\left\langle\mathbf{G}^{\prime}|\hat{\mathrm{W}}| \mathbf{G}\right\rangle \int \mathrm{d} \mathbf{r}\langle\mathbf{G} \mid \mathbf{r}\rangle\left\langle i_{\sigma} \mid \mathbf{r}\right\rangle\left\langle\mathbf{r} j_{\sigma}\right\rangle
\end{aligned}
$$

Here, $i, j$ index KS orbitals. The matrix elements of the Coulomb operator in reciprocal space, $\left\langle\mathbf{G}^{\prime}|\hat{W}| \mathbf{G}\right\rangle=\frac{1}{\Omega} \frac{4 \pi}{\mathbf{G}^{2}} \delta\left(\mathbf{G}-\mathbf{G}^{\prime}\right)$, exhibit an integrable divergence at $\mathbf{G}=0 .{ }^{32}$ In practice, the plane wave/pseudopotential formalism relies on a discrete representation of points in direct and reciprocal space, and the integrals become sums associated with discrete Fourier transforms. The divergence term becomes problematic in this discrete form, and the Coulomb operator has to be replaced by a suitable generalization $\hat{\Phi}$ :

$$
\begin{gathered}
E_{x}^{\mathrm{HFX}}=\sum_{\sigma} \sum_{i} \sum_{j \geq i} \int \mathrm{d} \mathbf{r}^{\prime}\left\langle j_{\sigma} \mid \mathbf{r}^{\prime}\right\rangle\left\langle\mathbf{r}^{\prime} \mid i_{\sigma}\right\rangle \int \mathrm{d} \mathbf{G}^{\prime}\left\langle\mathbf{r}^{\prime} \mid \mathbf{G}^{\prime}\right\rangle \times \\
\int \mathrm{d} \mathbf{G}\left\langle\mathbf{G}^{\prime}|\hat{\Phi}| \mathbf{G}\right\rangle \int \mathrm{d} \mathbf{r}\langle\mathbf{G} \mid \mathbf{r}\rangle\left\langle i_{\sigma} \mid \mathbf{r}\right\rangle\left\langle\mathbf{r} \mid j_{\sigma}\right\rangle
\end{gathered}
$$

In the generalized matrix element $\left\langle\mathbf{G}^{\prime}|\hat{\Phi}| \mathbf{G}\right\rangle$, the offending divergence is screened by a suitable function $\chi$ :

$$
\left\langle\mathbf{G}^{\prime}|\hat{\Phi}| \mathbf{G}\right\rangle= \begin{cases}\frac{1}{\Omega} \frac{4 \pi}{\mathbf{G}^{2}} \delta\left(\mathbf{G}-\mathbf{G}^{\prime}\right) & \text { for } \mathbf{G} \neq 0 \\ \chi(0) \delta\left(\mathbf{G}^{\prime}\right) & \text { for } \mathbf{G}=0\end{cases}
$$


where $\Omega$ denotes the supercell volume and $\hat{\Phi}$, like $\hat{W}$, is diagonal in $\mathbf{G}$.

In the initial treatment proposed by Gygi and Baldereschi, ${ }^{32}$ the screening term $\chi$ is obtained numerically. An auxiliary function which exhibits the same singularity as the problematic term is added to and subtracted from the Coulomb operator, and the screening is given by the difference of the discrete representation of the auxiliary function as a sum over $\mathbf{G}$ and its analytical integral over a continuous range $\mathbf{Q}$. Due to their particular choice of $\chi$, the approach could not be applied to $\Gamma$-point sampling due to its poor convergence with respect to the number of k-points and simulation supercell size. In the following, we base our treatment on the scheme subsequently developed by Broqvist et al. ${ }^{33}$ which-in contrast to the initial approach by Gygi and Baldereschi-selects an auxiliary function $f(\mathbf{Q})$ that converges rapidly toward $1 / \mathbf{Q}^{2}$ :

$$
f(\mathbf{Q})=\frac{\mathrm{e}^{-\gamma Q^{2}}}{Q^{2}}
$$

The $\mathbf{G}=0$ term is then given by the residual difference between the integral and discrete sum over the auxiliary function:

$$
\begin{gathered}
\chi(0)=\frac{1}{2 \pi^{2}} \int_{\mathbf{Q}} f(\mathbf{Q}) \mathrm{d} \mathbf{Q}-\frac{4 \pi}{\Omega} \sum_{\mathbf{G} \neq 0} f(\mathbf{G}) \\
=\lim _{\gamma \rightarrow 0}\left[\frac{1}{\sqrt{\pi \gamma}}-\frac{4 \pi}{\Omega} \sum_{\mathbf{G}} \frac{\mathrm{e}^{-\gamma \mathbf{G}^{2}}}{\mathbf{G}^{2}}\right]
\end{gathered}
$$

where the second term (the discrete sum) is obtained numerically. This results in a more rapid convergence with respect to the size of the supercell, and the scheme can therefore be applied in $\Gamma$-point only calculations.

Although a non-divergent analytical description for the $\mathbf{G}=0$ component is found for screened hybrids (it is simply $\pi / \Omega^{2} \mu^{2}$ ), its accuracy depends on the size of the periodic supercell. If $\mu$ is small, the potential does not decay sufficiently rapidly with respect to the number of $\mathbf{G}$-vectors and a treatment analogous to the non-screened Coulomb operator has to be used. ${ }^{33}$ Broqvist et al. have therefore suggested to treat the short-range exact exchange analogously to the full Coulomb operator:

$$
\left\langle\mathbf{G}^{\prime}\left|\hat{\Phi}_{\mathrm{sr}}\right| \mathbf{G}\right\rangle= \begin{cases}\frac{1}{\Omega} \frac{4 \pi}{\mathbf{G}^{2}}\left[1-\mathrm{e}^{-G^{2} / 4 \mu^{2}}\right] \delta\left(\mathbf{G}-\mathbf{G}^{\prime}\right) & \text { for } \mathbf{G} \neq 0 \\ \tilde{\chi}(\mu) \delta\left(\mathbf{G}^{\prime}\right) & \text { for } \mathbf{G}=0\end{cases}
$$

Using the same auxiliary function as for the full Coulomb potential, the resulting $\mathbf{G}=0$ term for the short-range screened exchange then reads:

$$
\tilde{\chi}(\mu)=\chi(0)-\chi\left(\frac{1}{4 \mu^{2}}\right)
$$

where $\mu$ is the range separation parameter.

Based on this treatment, the singularity correction for the CAM is easily found by using the identity $\operatorname{erf}(x)+\operatorname{erfc}(x)=1$ and introducing the parameters $\alpha$ and $\beta$. We write for the longrange components:

$$
\left\langle\mathbf{G}^{\prime}\left|\hat{\Phi}_{\mathrm{lr}}\right| \mathbf{G}\right\rangle= \begin{cases}\frac{1}{\Omega} \frac{4 \pi}{\mathbf{G}^{2}}\left[\alpha+\beta \mathrm{e}^{-G^{2} / 4 \mu^{2}}\right] \delta\left(\mathbf{G}-\mathbf{G}^{\prime}\right) & \text { for } \mathbf{G} \neq 0 \\ \bar{\chi}(\mu, \alpha, \beta) \delta\left(\mathbf{G}^{\prime}\right) & \text { for } \mathbf{G}=0\end{cases}
$$

where the $\mathbf{G}=0$ term is simply a sum of the terms due to the full and the screened Coulomb potential, weighted by the attenuation parameters $\alpha$ and $\beta$ :

$$
\bar{\chi}(\mu, \alpha, \beta)=\alpha \chi(0)+\beta \chi\left(\frac{1}{4 \mu^{2}}\right)
$$

\section{IMPLEMENTATION}

Some xc functionals make use of a linear combination of different GGA exchange contributions, such as XLYP ${ }^{34}$ (using $72.2 \% \mathrm{~B} 88^{35}$ and $34.7 \% \mathrm{PW}^{36} 1^{36}$ exchange) or the well-known B3LYP (using $80 \%$ LDA, $72 \%$ of the B88 gradient correction term and $20 \%$ exact exchange). Accordingly, the usage of the CAM does not have to be intrinsically limited to a single type of exchange functional (as it is the case for the most prominent CAM-B3LYP and LC-PBE0, where B88 and PBEx are attenuated, respectively). With respect to all possible combinations, our implementation achieves maximum flexibility in the choice of exchange functional by writing the exchange enhancement factor as a sum over individual contributions:

$$
K_{\sigma}^{x}=\sum_{f}^{N} c_{f} K_{\sigma}^{f}
$$

where $K_{\sigma}^{f}$ denotes any GGA exchange-functional and $c_{f}$ are the corresponding weights of a total of $N$ exchange functionals: $\sum_{f}^{N} c_{f}=1$. The attenuation is then applied to $K_{\sigma}^{x}$ after all $N$ contributions have been added up. This makes it possible to consistently apply the CAM to any arbitrary combination of LDA and GGA exchange functionals, creating a custom "à-la-carte” xc functional.

Three terms are needed for the propagation of a KS wave function with a GGA description:

$$
\begin{aligned}
& E[\rho]=-\frac{1}{2} \sum_{\sigma} \int \eta\left[K_{\sigma}^{x}\right] K_{\sigma}^{x}\left[\rho_{\sigma}, \nabla \rho_{\sigma}\right] \rho_{\sigma}^{4 / 3}(\mathbf{r}) \mathrm{d} \mathbf{r} \\
& =-\frac{1}{2} \sum_{\sigma} \int \epsilon\left[\rho_{\sigma}(\mathbf{r})\right] \mathrm{d} \mathbf{r}
\end{aligned}
$$

$V_{1}^{x}=\frac{\delta \epsilon[\rho]}{\delta \rho_{\sigma}}$

$$
V_{2}^{x}=\frac{\delta \epsilon[\rho]}{\delta\left|\nabla \rho_{\sigma}\right|}
$$

where we have introduced $\eta$ as the attenuation function. For functionals that are derived for the closed-shell case or use a different definition of the enhancement factor, e.g., $E[\rho]=$ $\int \rho(\mathbf{r}) F[\rho] \mathrm{d} \mathbf{r}$, the spin-dependent exchange enhancement factor $K_{\sigma}^{x}$ is easily obtained from $F[\rho]$ or $K_{\alpha \beta}^{x}$ using the spinscaling relations.

All derivatives can be efficiently calculated by making extensive use of the chain rule and by transiently storing frequently used terms (notably $\delta K_{x} / \delta \rho, \delta K_{x} / \delta \nabla \rho$ ) on the stack. In order to make further performance gains, certain powers of the density and the gradient $\left(\rho, \rho^{4 / 3}, \rho^{1 / 3},|\nabla \rho|, \nabla \rho^{2}\right)$ are precomputed on a per-grid-point basis and reused in the calculation of $K_{\sigma}^{f}$ and the attenuation function, thus avoiding repetitive, unnecessary operations. The implementation makes use of procedure pointers in order to facilitate the choice of functional. At the beginning of every run, the procedure pointer denoting $K_{\sigma}^{f}$ is set to the 
selected exchange functional, no (explicit) if-construct is therefore necessary when looping over grid points. The new algorithm reaches an asymptotic speedup of $20 \%$ when calculating the LDA and GGA contributions with respect to the

SUBROUTINE calculate_xc_energy (rho,grad,E_x)

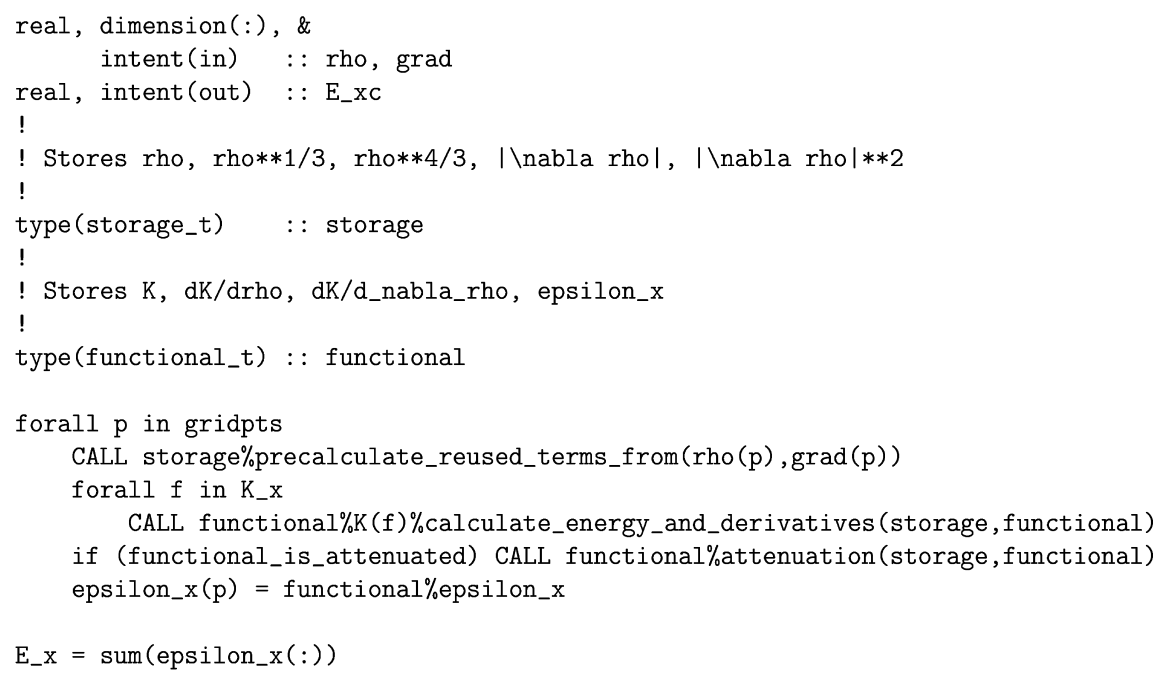

END SUBROUTINE calculate_xc_energy

\section{TEST SET AND COMPUTATIONAL DETAILS}

4.1. Description of the Test Set. Basic convergence tests with respect to basis set and supercell size were carried out on a single, isolated water molecule.

A test set for excitation energies was assembled comprising the molecules mentioned before and depicted in Figure 1. The set includes the original test systems by Peach et al., ${ }^{9}$ augmented with other systems where range-separation is known to be of importance: $p$-nitroaniline, 7-azaindole, the AT nucleobase dimer, and the retinal protonated Schiff base.

For the sake of comparison with localized basis sets, five of those systems were selected. The model dipeptide represents a typical system where CAM-B3LYP can successfully be applied: Peach et al. found that a GGA (PBE) yields an inaccurate state ordering of the CT excitations, whereas B3LYP and CAMB3LYP reproduce the ordering of the reference values. However, B3LYP is known to underestimate the energies of the $n_{1} \rightarrow \pi_{2}^{*}$ and $\pi_{1} \rightarrow \pi_{2}^{*} \mathrm{CT}$ excitations by up to $1.7 \mathrm{eV}$, which are both reproduced by CAM-B3LYP with a reasonable accuracy of about $0.2 \mathrm{eV}$. The spectrum of $p$-nitroaniline has also been reported to be reasonably predicted using CAM-B3LYP, ${ }^{24}$ and although the charge separation in the first CT state was reported to be overestimated, we have used $p$-nitroaniline as another probe known to benefit from range-separation. Naphthalene served as the most simple example of an acene and another notable example of the influence of range separation: Whereas the ${ }^{1} B_{2 u}$ and ${ }^{1} B_{3 u}$ states are inverted when using both PBE and B3LYP, only CAM-B3LYP recovers the correct state ordering. However, the reported excitation energies for the two lowest optically allowed singlet transitions deviate from the reference by about $+0.21 \mathrm{eV}$ for ${ }^{1} B_{3 u}$ and $-0.16 \mathrm{eV}$ for ${ }^{1} B_{2 w}$, which results in a considerable underestimation of the state separation. The separation is reported to improve for larger acenes (anthracene, tetracene etc.); we have therefore chosen naphthalene as the most critical and sensitive compound to assess our implementation. An example where CAM-B3LYP (narrowly) fails to deliver a quantitatively correct description of states is given by 7 -azaindole. previous standard implementation, which obtained LDA and GGA contributions for each functional from separate drivers and used extensive if-constructs to select among the functionals at every individual gridpoint. 
when using PBE. The $\beta$-dipeptide therefore serves as yet another example in which CAM-B3LYP does not work even on a quantitative level, probably constituting the hardest test case for new range-separated functionals. Finally, in order to verify a possible overall gain of accuracy, the MAE of both CAM-B3LYP and the customized functional is compared for the complete test set by Peach et al. ${ }^{9}$

4.2. Computational Setup. Calculations using Gaussian basis sets were either carried out using DALTON 2016 ${ }^{40}$ ( $p$-nitroaniline, the retinal protonated Schiff base and naphthalene) or the Gaussian09 suite of programs ${ }^{41}$ (the remaining molecules) using Dunning's correlation-consistent basis sets. ${ }^{42}$ All calculations employing a Slater basis set ${ }^{43}$ were carried out using ADF. ${ }^{44-46}$

The structures for the dipeptides, DMABN, and naphthalene were taken from the database published by Peach et al. The structure of the retinal protonated Schiff base is an arbitrarily chosen snapshot extracted from molecular dynamics simulations at $300 \mathrm{~K}$, whereas the structure of $p$-nitroaniline was optimized using the aug-cc-pVTZ basis and the B3LYP xc functional. Structural optimizations for the remaining molecules were performed using the Gaussian suite of programs, following the published protocols of existing benchmarks for the AT base pair ${ }^{15}$ and 7 -azaindole. ${ }^{16}$ Excitation energies, where not quoted from the literature, were calculated using the Tamm-Dancoff approximation to TD-DFT ${ }^{47}$ and the cc-pVDZ, aug-cc-pVDZ, and d-aug-cc-pVDZ basis sets, respectively.

The new xc driver was implemented in a development version of $\mathrm{CPMD}^{30}$ (successor of version 4.1). The KS orbitals were expanded in plane waves contained in an orthorhombic supercell of varying dimensions and using either Martins-Troullier $(\mathrm{MT})^{48}$ or Goedecker-Teter-Hutter $(\mathrm{GTH})^{49}$ pseudization of the atomic core orbitals (the respective values for the energy cutoff are given in the results section; the supercell size for every system is available in the Supporting Information). Following standard practice for hybrid functionals, BLYP pseudopotentials were used for CAM-B3LYP calculations, and OLYP pseudopotentials were used for calculations with CAM-O3LYP. The density was expanded with a 4 times greater cutoff value than the one adopted for the orbitals. The Poisson equations for the isolated systems were solved using the algorithm of Martyna and Tuckerman. ${ }^{50}$ All calculations made use of an atomic wave function initialization using distributed Lanczos, ${ }^{51}$ the new distributed linear algebra algorithm by Bekas and Curioni ${ }^{52}$ and the "new" exact exchange driver by Weber et al.; ${ }^{31}$ the cutoff in the calculation of the Fock exchange energy was not changed with respect to the standard values for orbitals and density.

\section{RESULTS AND DISCUSSION}

5.1. Convergence of Eigenvalues. 5.1.1. HOMO-LUMO Gaps in a Plane Wave/Pseudopotential Basis. When implementing the singularity correction for (screened) hybrid functionals, Broqvist et al. ${ }^{33}$ have also assessed the convergence of the KS HOMO-LUMO gaps with respect to both the energy cutoff and the size of the supercell. In the following, we shall present a similar assessment on an isolated water molecule. Considering that the LUMO is very diffuse in this specific case, the HOMO-LUMO gap appears to be a sensitive measure of convergence.

When assessing the convergence with respect to the size of the simulation supercell, two scenarios have to be distinguished: In a periodic setup, the gap for an isolated system can only be reproduced when the molecule at the center of the cell is sufficiently far apart from its periodic images. When the
Poisson equations of the replicas are decoupled (and the requirements of the used Poisson solver appropriately met $^{50}$ ), the gap will converge with respect to the lowest G-vector components, which corresponds to increasingly longer-range components in real space as the supercell size increases. This may be especially important if the LUMO is very diffuse (we note that an unbound continuous state will only be appropriately described if the length of the simulation cell $l=\infty$ ). Hence, a change in cutoff value enhances the accuracy of the description by adding more rapidly oscillating, short-range components; the maximum "diffuseness" allowed is essentially governed by the choice of $l$ for the simulation cell.

Table 1 shows the gap obtained using the CAM-B3LYP xc functional for an isolated system contained in varying sizes of

Table 1. HOMO-LUMO Gap of a Water Molecule Calculated Using CAM-B3LYP and GTH Pseudopotentials at Increasing Energy Cutoffs and Varying Cubic Simulation Cell Lengths $l$ Using Periodic or Isolated System Boundary Conditions for Solving the Poisson Equation

\begin{tabular}{ccccccccc} 
& \multicolumn{2}{c}{$\Delta \epsilon_{i}[\mathrm{eV}]$, isolated system } & & \multicolumn{3}{c}{$\Delta \epsilon_{i}[\mathrm{eV}]$, periodic system } \\
\cline { 2 - 4 } \cline { 7 - 8 }$E_{\text {cut }}[\mathrm{Ry}]$ & $10 \AA$ & $20 \AA$ & $30 \AA$ & & $10 \AA$ & $20 \AA$ & $30 \AA$ \\
70 & 10.080 & 10.361 & 10.399 & & 10.105 & 10.362 & 10.399 \\
80 & 10.073 & 10.420 & 10.447 & & 10.100 & 10.420 & 10.448 \\
100 & 10.150 & 10.490 & 10.510 & & 10.177 & 10.491 & 10.509 \\
120 & 10.259 & 10.531 & 10.548 & & 10.284 & 10.532 & 10.548 \\
150 & 10.345 & 10.560 & 10.577 & & 10.367 & 10.561 & 10.577 \\
180 & 10.383 & 10.574 & 10.591 & & 10.404 & 10.575 & 10.591 \\
\hline
\end{tabular}

the simulation supercell using hard GTH pseudopotentials. Values for a fully periodic system are also given. The corresponding values for softer MT pseudopotentials are tabulated in the Supporting Information.

The gaps show convergence at 150 Ry for all systems. For a small cubic simulation supercell $(l=10 \AA)$, choosing a lower cutoff value of 100 Ry introduces a substantial error of $0.23 \mathrm{eV}$. A notable error is still present at 100 Ry even for the two larger simulation cells, but it becomes less relevant for practical purposes, since the maximal deviation of $<0.1 \mathrm{eV}$ lies below the typical accuracy of the functional itself. Whereas the values in the smallest of the supercells still have an error of about $0.1 \mathrm{eV}$ at a cutoff of $120 \mathrm{Ry}$, the corresponding values have converged in the 20 and $30 \AA$ supercells, with errors being lower than $0.05 \mathrm{eV}$, and full convergence is reached at $150 \mathrm{Ry}$ for all of the three supercells considered.

The convergence behavior is analogous to that observed for GGA or standard hybrid functionals once the simulation cell is of sufficient size: Changes in the gap are still substantial when increasing the length $l$ of the cubic simulation cell from 10 to $20 \AA$, with changes in the converged gap of $0.2 \mathrm{eV}$. The change in gap is within the usual numerical tolerance $(<0.05 \mathrm{eV})$ for a further extension to $30 \AA$, emphasizing again the importance of an appropriately large cell for the correct description of the system's LUMO.

The results for the fully periodic system (where the Poisson equations are not decoupled) show the same trend, with the only relevant difference with respect to the isolated system occurring in the $10 \AA$ A box. Still, these differences are lower than those observed when enlarging the supercell. Given the trends observed for the isolated system, this is most likely attributed to spurious interactions between periodic images at this intermolecular distance. The influence of these interactions on the gap supports the 
view that the requests on the Tuckerman-Martyna Poisson solver are not yet met either, since the simulation cell must span at least twice the spatial extent of the charge density. The strong changes in gaps when increasing the cutoff within the small simulation cell is hence due to an insufficient cell size for both the isolated and periodic system, resulting in an incorrect description of the electron density.

The same considerations hold for the gaps obtained with the softer MT pseudopotentials (cf. the Supporting Information), albeit convergence is achieved at lower cutoff values. The maximum deviation in the converged gaps with respect to GTH pseudization is very small, $\Delta \Delta \epsilon=0.02 \mathrm{eV}$, illustrating that the influence of the pseudization of the cusp on the gaps is negligibly low.

These results confirm that the use of the CAM in a plane wave/pseudopotential formalism does not introduce any convergence issues or additional restrictions, given that the size of the simulation supercell is chosen sufficiently large in order to accommodate the whole spectrum of $\mathbf{G}$-vectors that are required to span the range of the $\mu$-dependent switching function.

5.1.2. Comparison with Atom-Centered Basis Sets. Plane waves inherently contain diffuse components even at a low cutoff energy, and the character of the diffuse functions is restricted only by the size of the supercell; convergence is reached with respect to high-frequency components needed to describe the region around the pseudized core. In turn, the number of short-range components and the description of the orbitals around the nuclei can be systematically improved by using pseudopotentials of increasing hardness (which requires a simultaneous increase in the cutoff value). The complete basis set limit can therefore be systematically reached (once completeness holds with respect to the adopted pseudopotential).

The situation presents itself substantially different in atomcentered basis sets. In a Gaussian basis, the orbitals around the nuclei are well described, but for an accurate description of most molecular properties, the compact basis usually has to be enhanced by augmentation with long-range functions. When the KS orbitals are expanded in Gaussians, a single set of diffuse functions is often sufficient for routine applications. (A more realistic description of the cusp and a correct decay of the basis at long range can be obtained by resorting to a Slater-type basis.)

A comparison between plane wave and atom-centered basis set calculations can therefore reveal the influence of the longestrange components (described well within plane waves) and the explicit description of the orbitals around the nuclear cusp (reproduced well using Gaussian functions). In the following, we will compare the HOMO-LUMO gaps of the preceding section with the corresponding results obtained from various Gaussian basis sets of increasing accuracy. Additional tests were performed using a Slater-type basis in order to obtain a systematic analysis with respect to the cusp condition. The results are shown in Table 2.

When increasing the size of the basis from double to hextuple zeta, changes in the gap are considerable for the non-augmented basis sets, spanning a range of a total of $0.9 \mathrm{eV}$. Once a single diffuse function is included, the gaps become much more uniform, with a difference between aug-cc-pVDZ and aug-cc-pVTZ of only $25 \mathrm{meV}$. When increasing zeta to $\zeta=6$, the gap fluctuates within a negligible range of $3 \mathrm{meV}$. Adding more diffuse functions never changes the gap by more than $5 \mathrm{meV}$. The aug-ccpVDZ basis can therefore be considered sufficiently accurate when calculating HOMO-LUMO gaps with CAM-B3LYP.

The trend is very similar for a Slater-type basis, where we have only included single-zeta values for comparison. As for the
Table 2. HOMO-LUMO Gap of a Water Molecule Calculated Using Atom-Centered Basis Sets Augmented with a Varying Number of Diffuse Functions

\begin{tabular}{lrccc} 
& \multicolumn{4}{c}{ no. of augmentation functions } \\
\cline { 2 - 5 } & \multicolumn{4}{c}{ Gaussian Basis } \\
cc-pVDZ & 9.832 & 10.679 & 10.667 & 10.666 \\
cc-pVTZ & 10.387 & 10.702 & 10.696 & 10.695 \\
cc-pVQZ & 10.576 & 10.705 & 10.670 & 10.700 \\
cc-pV5Z & 10.691 & 10.705 & 10.700 & 10.700 \\
cc-pV6Z & 10.714 & 10.704 & 10.700 & 10.700 \\
& & & & \\
SZ & 17.300 & 10.711 & \\
DZ & 12.318 & 10.631 & \\
TZ & 11.310 & 10.639 & \\
\hline
\end{tabular}

Gaussian basis set, the omission of diffuse functions leads to an insufficient description of the gap. However, as soon as a single set of diffuse functions is included, the gap again converges rapidly. The change from augmented double to triple zeta is only about $8 \mathrm{meV}$. Even an augmented single-zeta basis seems to yield a surprisingly accurate gap, with the difference being only $0.08 \mathrm{eV}$ with respect to the augmented triple-zeta basis.

While the difference between the converged gaps in plane waves and the corresponding values in a Gaussian basis is about $0.1 \mathrm{eV}$, it is only $0.03 \mathrm{eV}$ when compared to a Slater-type basis (augmented triple- $\zeta$ vs GTH/180 Ry/30 Å cell). Even though the orbitals are pseudized around the core, the plane wave/ pseudopotential approach yields results which are virtually indistinguishable from all-electron calculations with an atomcentered basis. The slightly larger deviation with respect to the Gaussian basis may be attributed to differences in the longrange decay and the description of the cusp, but they still lie well within what is usually deemed chemical accuracy.

5.2. Excitation Energies. The most frequent use of Coulomb-attenuated functionals is the description of excited states (which is influenced by the accuracy of the KS eigenvalues examined in the previous section through the linear response equations). We therefore conclude the assessment of our implementation of the CAM in plane waves by comparing the results to excited-state energies obtained using Gaussian bases. In line with the trends observed in the preceding section, only the cc-pVDZ basis was considered, which was augmented with a different number of diffuse functions. The results are depicted in Table 3.

The excitation energies were computed for the set of molecules introduced above, which apart from DMABN all contain transitions with CT or Rydberg character. Since the plane wave/pseudopotential implementation of LR-TD-DFT in the CPMD code is limited to the Tamm-Dancoff approximation (TDA), the electronic spectra in a Gaussian basis were obtained within the same approximation for the sake of comparison. While it has been found that high-overlap singlet transitions can be substantially affected by the use of the TDA, it has also been reported that the results obtained from the TDA compare more favorably to high-level reference values than those obtained from full TD-DFT. ${ }^{53}$ This effect can be understood in terms of the triplet stability measure, the corresponding values for a subset of the molecules considered here have been reported and discussed in ref 53.

For the dipeptide, DMABN and 7-azaindole, the results of the singly augmented Gaussian basis sets already exhibit a 
Table 3. Excitation Energies of the First Two to Four Excited Singlet States Obtained for Various Systems and Basis Sets Using LR-TD-DFT and the CAM-B3LYP xc Functional within the Tamm-Dancoff Approximation ${ }^{a}$

\begin{tabular}{|c|c|c|c|c|c|c|}
\hline \multirow[b]{2}{*}{ state } & \multicolumn{4}{|c|}{ CAM-B3LYP } & \multirow{2}{*}{$\frac{\text { CAM-O3LYP }}{\mathrm{MT} / 80 \mathrm{Ry}}$} & \multirow[b]{2}{*}{ ref } \\
\hline & cc-pVDZ & aug-cc-pVDZ & d-aug-cc-pVDZ & $\mathrm{MT} / 80 \mathrm{Ry}$ & & \\
\hline \multicolumn{7}{|c|}{ Naphthalene ${ }^{54}$} \\
\hline$S_{1}$ & - & 4.66 & - & 4.65 & 4.63 & 4.46 \\
\hline $\mathrm{S}_{2}$ & 4.73 & 4.86 & 4.86 & 4.79 & 4.88 & 4.88 \\
\hline \multicolumn{7}{|c|}{$\mathrm{DMABN}^{55}$} \\
\hline$S_{1}$ & 4.91 & 4.76 & 4.76 & 4.77 & 4.68 & 4.25 \\
\hline $\mathrm{S}_{2}$ & 5.29 & 4.98 & 4.96 & 4.95 & 4.81 & 4.56 \\
\hline \multicolumn{7}{|c|}{7 -azaindole ${ }^{56}$} \\
\hline$S_{1}$ & $5.19^{1} L_{a}$ & $5.06{ }^{1} L_{a}$ & $5.05{ }^{1} L_{a}$ & $5.03{ }^{1} L_{a}$ & $5.05{ }^{1} L_{b}$ & $4.22{ }^{1} L_{b}$ \\
\hline $\mathrm{S}_{2}$ & $5.24{ }^{1} L_{b}$ & $5.08{ }^{1} L_{b}$ & $5.08{ }^{1} L_{b}$ & $5.07{ }^{1} L_{b}$ & $5.09{ }^{1} L_{a}$ & $4.49{ }^{1} L_{a}$ \\
\hline $\mathrm{S}_{3}$ & 5.36 & 5.32 & 5.32 & 5.33 & 5.35 & 5.27 \\
\hline \multicolumn{7}{|c|}{ Dipeptide $^{26}$} \\
\hline$S_{1}$ & 5.67 & 5.67 & 5.67 & 5.72 & 5.71 & 5.62 \\
\hline $\mathrm{S}_{2}$ & 5.92 & 5.89 & 5.88 & 5.93 & 5.91 & 5.79 \\
\hline $\mathrm{S}_{3}$ & 7.09 & 6.24 & 6.24 & 6.20 & 6.16 & 7.18 \\
\hline $\mathrm{S}_{4}$ & 7.33 & 6.55 & 6.51 & 6.44 & 6.38 & 8.07 \\
\hline \multicolumn{7}{|c|}{$p$-nitroaniline $\mathrm{e}^{57}$} \\
\hline$S_{1}$ & 4.02 & 4.00 & 4.00 & & 4.19 & 4.12 \\
\hline $\mathrm{S}_{2}$ & 4.57 & 4.53 & 4.53 & 4.54 & 4.59 & 4.66 \\
\hline $\mathrm{S}_{3}$ & & 4.57 & & & 4.69 & 4.68 \\
\hline $\mathrm{S}_{4}$ & 4.79 & 4.87 & 4.87 & 4.87 & 4.92 & 4.75 \\
\hline
\end{tabular}

${ }^{a}$ Values for a customized CAM-O3LYP functional are indicated, too. Literature reference values from high-level wavefunction calculations are given where available. In $p$-nitroaniline, the first forbidden transition is not predicted in a plane-wave basis when using CAM-B3LYP; its oscillator strength is $f=0$ in the other calculations. The character of the transition is indicated whenever it is not consistent within different methods. Reference values for naphthalene, DMABN and the dipeptide correspond to those adopted by Peach et al. .

negligible difference to those obtained with the plane wave/ pseudopotential approach. Neither the character nor the energetics of the transitions do change when using a doubly augmented Gaussian basis. Both Gaussians and plane waves yield the same ordering of states and differences in the excitation energies are smaller than $0.05 \mathrm{eV}$; i.e., they lie within a range that we have previously considered as converged. The small deviations may be attributed to both the pseudization of the orbitals in plane waves, as well as the limited spatial extent of a localized atom-centered basis, along with its predefined decay properties.

The situation is different in naphthalene and $p$-nitroaniline. In naphthalene, the $S_{1}$ state dominated by a HOMO-1 $\rightarrow$ LUMO transition is only predicted using plane waves or the singly augmented atom-centered Gaussian basis. However, the energetics of the $S_{2}$ state (mainly HOMO $\rightarrow$ LUMO) is consistent between the singly and doubly augmented Gaussian basis. The excitation energies agree well between our plane wave implementation and aug-cc-pVDZ.

The basis-set dependency issue becomes more involved in the case of $p$-nitroaniline. Whereas the $S_{2} \pi \rightarrow \pi^{*}$ transition is predicted in all cases, the $S_{3} n \rightarrow \pi^{*}$ state at $4.68 \mathrm{eV}$ only appears when one single diffuse function is used. Once a further set of diffuse functions is included, the $S_{3} n \rightarrow \pi^{*}$ transition disappears again. It can therefore be concluded that in the case of $p$-nitroaniline, the description of the $S_{3}$ state using CAMB3LYP is particularly sensitive with respect to the choice of basis. Since the $S_{3}$ transition disappears once the basis is enlarged, this indicates that the (quantitatively correct) prediction using augcc-pVDZ is a mere artifact, and that CAM-B3LYP is not properly able to describe the excitations in the limit of a complete basis. This view is further supported by the results obtained in plane waves, where the $S_{3}$ state is absent: The ordering of the states obtained at a cutoff of 120 Ry coincides with the one obtained using a doubly augmented Gaussian basis. It has to be noted that the $S_{1}$ state has an oscillator strength $f=0$, and that it is not predicted when using plane waves.

Overall, the results obtained using a plane wave pseudopotential approach are in excellent agreement with the ones obtained with all-electron calculations in a Gaussian basis set. If any of the orbitals included in the transitions of interest is highly diffuse, plane waves fare better than a singly augmented Gaussian basis. There is no indication that the presence (or absence) of pseudization has any (relevant) influence on the spectra, with the remaining differences between the doubly augmented Gaussian basis and the plane waves being vanishingly small. Although the excitation energies usually do not change considerably when approaching the diffuse limit, the character and number of states may, which can be important for applications such as excited-state dynamics. The observations made in $p$-nitroaniline further stress the importance of a sufficiently large basis, since seemingly correct predictions may be an artifact due to an incomplete basis. Only the use of a very diffuse Gaussian basis set or plane waves reveal that CAM-B3LYP does not properly predict one of the transitions. Given that most standard applications of Coulomb-attenuated functionals use only a single set of augmentation functions, plane waves hence offer the advantage of converging much more rapidly toward the basis set limit, thanks to their inherently diffuse character. This is further 
illustrated by the haphazard description of the $S_{1}$ state in naphthalene, which is easily recovered in plane waves.

5.3. Performance of New Customized "à-la-Carte" Coulomb-Attenuated Functionals. Our generalized implementation of the CAM renders adjustments to the established $\mathrm{xc}$ functionals straightforward, for instance by using different range-separation parameters $\alpha, \beta$, or by changing $\mu$ (commonly referred to as $\gamma$-tuning). The flexible form of $K_{\sigma}^{x}$ also opens the distinct possibility of assembling new long-range corrected functionals based on simple physicochemical considerations.

CAM-B3LYP has become a valuable tool in the calculation of excited states, as illustrated by the substantial improvements of the excitation energies with respect to a simple GGA published in the aforementioned studies. Still, the values in Table 3 reveal that not all transitions may be accurately captured quantitatively or qualitatively. The basis-set sensitivity of the excitations in $p$-nitroaniline is more of a practical issue, since reliable values may still be recovered in an accurately large basis, although at a substantial computational cost. However, the wrong ordering of states in 7-azaindole is one example that can only be overcome by resorting to generally less widely applicable xc functionals such as LC-BLYP, with a similar situation occurring in the $\beta$-dipeptide. The orbital localization problems in the AT base pair represent a further challenge. Such problems are often attributed to stem from an imbalance between short- and longrange exchange. The usual remedy to this weakness is the use of LC-BLYP, which fares worse for the systems for which CAMB3LYP excels, but offers a more reasonable description of compounds where the ordering of CT or Rydberg-states proves to be inaccurate. In the following, we have attempted to construct a functional that is sufficiently accurate for both systems where CAM-B3LYP is of satisfactory accuracy, as well as for the few CT systems where the functional has its known weaknesses.

A modified version of CAM-B3LYP with $80 \%$ exact exchange at long range was reported to yield eigenvalue differences closer to LC-BLYP, but performed worse for other properties where the "standard" CAM-B3LYP yields accurate results. ${ }^{11}$ LC-BLYP includes no exact exchange at shortest range, whereas CAMB3LYP still includes $19 \%$, a value close to the $20 \%$ used in standard B3LYP. A suitable compromise between LC-BLYP and CAM-B3LYP could therefore lie in attenuating an existing hybrid functional with more GGA exchange at short range, and less at long range. Following the CAM-B3LYP approach, the short-range contribution of the exact exchange should lie close to the value used in the conventional hybrid to ensure proper balance at short range.

Handy's OPTX ${ }^{58}$ functional in conjecture with Lee-YangParr correlation ${ }^{59}$ has on several occasions been shown to be superior to Becke's 1988 exchange functional, ${ }^{60-63}$ and hybrids including OPTX such as O3LYP ${ }^{58}$ include a lower percentage of exact exchange than the famous B3LYP while retaining comparable accuracy. We therefore assumed a Coulomb-attenuated version of O3LYP with $80 \%$ exact exchange at long range (as in the CAM-B3LYP declination in ref 11 ) and only $11 \%$ at short range (as in the O3LYP-hybrid) to offer the same benefits as LC-BLYP or CAM-B3LYP with $80 \%$ exact exchange at long range, but with an improved description of the short-range region due to the inclusion of the more accurate OPTX.

The performance of the CAM-O3LYP functional on the test set used in the previous chapter, including the problematic 7-azaindole, is summarized in Table 3. In the dipeptide, much like the retinal protonated Schiff base, CAM-O3LYP yields virtually indistinguishable results from CAM-B3LYP, with a maximum deviation in $S_{4}$ of $0.06 \mathrm{eV}$. The $S_{1}$ and $S_{2}$ states are therefore accurately described, whereas the deviations for $S_{3}$ and $S_{4}$ remain too large for practical applications in both of the functionals. We should note that the good agreement between CAM-B3LYP and reference values reported by Peach et al. were based on values obtained in a basis without diffuse functions and without the TDA; the higher energies of the CT states we report for the cc-pVDZ basis are in line with their results. With CAM-O3LYP, the state ordering in 7-azaindole is now correctly reproduced, although the absolute errors are still considerable, with the energetic difference between the ${ }^{1} L_{a}$ and ${ }^{1} L_{b}$ states being too low. (This is also observed for CAM-B3LYP and may be attributed to the use of the TDA, since in general, the energy difference between the two states becomes larger if the TDA is not employed.) In $p$-nitroaniline, the spectrum substantially improves with the use of CAM-O3LYP, due to an improved description of the $S_{3}$ state, which is now predicted even when approaching the basis set limit. For systems in which a classical hybrid functional is preferable to CAM-B3LYP, such as DMABN, the error due to CAM-O3LYP is comparable to the one of CAMB3LYP, even slightly smaller in the case of the $S_{2}$ state.

Table 4 shows the excitation energies for a structure of the retinal protonated Schiff base from a molecular dynamics

Table 4. Comparison of Excitation Energies between CAM-B3LYP and CAM-O3LYP for a Structure of the Retinal Protonated Schiff Base Obtained from a Molecular Dynamics Snapshot ${ }^{a}$

$\begin{array}{ccc}\text { state } & \text { CAM-B3LYP } & \text { CAM-O3LYP } \\ \mathrm{S}_{1} & 2.66 & 2.63 \\ \mathrm{~S}_{2} & 4.13 & 4.25 \\ \mathrm{~S}_{3} & 4.97 & 4.95 \\ \mathrm{~S}_{4} & 5.12 & 5.08\end{array}$

${ }^{a}$ CAM-B3LYP is known to give good agreement with respect to highlevel wavefunction methods for this molecule. ${ }^{17,38}$

snapshot calculated with both CAM-B3LYP and CAM-O3LYP. Since CAM-B3LYP has been shown to yield very accurate excitation energies in this system, this can serve as an additional benchmark for the accuracy of our new functional. Indeed, CAM-O3LYP yields virtually indistinguishable excitation energies.

The remaining "problematic cases", the AT base pair and the $\beta$-dipeptide are presented in Table 5, where excitation energies obtained from CAM-O3LYP and CAM-B3LYP are compared to reference values.

For one of the two notorious cases, CAM-O3LYP outperforms CAM-B3LYP on a qualitative level: The spectrum of the AT base pair is qualitatively correctly reproduced, with the HOMO lying on adenine rather than thymine, in contrast to CAM-B3LYP results. Overall, both functionals capture the energetics of all but the $S_{1}$ state accurately, but CAM-O3LYP gives a correct theoretical description of the orbital localization. In the $\beta$-dipeptide there is no improvement with respect to CAM-B3LYP, but the qualitative and quantitative behaviors are again very similar.

Overall, CAM-O3LYP shows identical or superior performance to CAM-B3LYP for all of the excitations; it appears to be more versatile in the description of systems that require a larger percentage of exact exchange at long range, where it is able to remedy some of the pitfalls encountered with CAM-B3LYP. These are systems where LC-BLYP has typically been used so far. While the mean absolute error (MAE) over all the 
Table 5. Comparison of Excitation Energies between CAMB3LYP, CAM-O3LYP, and Reference Values for Some Typical Usage Cases of Range-Separated Functionals Known To Be Problematic When Treated with CAM-B3LYP ${ }^{a}$

$\begin{array}{llcl}\text { state } & \text { CAM-B3LYP } & \begin{array}{c}\text { CAM-O3LYP } \\ \text { AT Base Pair }{ }^{64}\end{array} \\ \mathrm{~S}_{1} & 5.25 & 5.24 & 4.94 \\ \mathrm{~S}_{2} & 5.26 & 5.29 & 5.21 \\ \mathrm{~S}_{3} & 5.36 & 5.40 & 5.40 \\ \mathrm{~S}_{4} & 5.38 & 5.45 & 5.47 \\ & & & \\ & & \beta \text {-dipeptide } & \\ \mathrm{S}_{1} & 5.69 n_{1} \rightarrow \pi_{1}^{*} & 5.66 n_{1} \rightarrow \pi_{1}^{*} & 5.10 n_{2} \rightarrow \pi_{2}^{*} \\ \mathrm{~S}_{2} & 5.76 n_{2} \rightarrow \pi_{2}^{*} & 5.74 n_{2} \rightarrow \pi_{2}^{*} & 5.40 n_{1} \rightarrow \pi_{1}^{*} \\ \mathrm{~S}_{3} & 6.06 \pi_{1} \rightarrow \pi_{2}^{*} & 6.00 \pi_{1} \rightarrow \pi_{2}^{*} & 7.99 \pi_{1} \rightarrow \pi_{2}^{*} \\ \mathrm{~S}_{4} & 6.14 n_{1} \rightarrow \pi_{2}^{*} & 6.01 n_{1} \rightarrow \pi_{2}^{*} & 9.13 n_{1} \rightarrow \pi_{2}^{*}\end{array}$

${ }^{a}$ The character of the transition is given where it differs with respect to the reference. The reference values for the $\beta$-dipeptide correspond to those adopted by Peach et al.; ${ }^{9}$ remaining values for molecules included therein and not explicitly mentioned so far are tabulated in the Supporting Information.

molecules considered here is about $\mathrm{MAE}=0.65 \mathrm{eV}$ for CAMB3LYP, it improves for CAM-O3LYP, where MAE $=0.55 \mathrm{eV}$.

This demonstrates that based on simple considerations, a new Coulomb-attenuated functional can be constructed. CAM-O3LYP predicts qualitatively correct state ordering where CAM-B3LYP fails, and in these systems yields excitation energies similar to the latter. With CAM-O3LYP, it is therefore possible to cover a larger range of systems than with CAM-B3LYP with slightly improved accuracy. This is further reflected by the MAE of both functionals, which is about $0.1 \mathrm{eV}$ lower for the CAM-O3LYP presented here. For other delicate systems and specific problems, our implementation offers the possibility of adapting existing xc functionals at hand, or to assemble entirely new Coulombattenuated functionals.

\section{CONCLUSIONS}

We have presented a new, efficient and fully flexible implementation of Coulomb-attenuated functionals in the plane wave/ pseudopotential code CPMD which allows for a customized composition of exchange-correlation functionals. On the base of a comprehensive test suite, we could demonstrate that the results obtained within the plane wave/pseudopotential framework do not significantly deviate with respect to results obtained in all-electron calculations with Gaussian bases. The results indicate that the complete basis set limit is more easily reached in plane waves, and that the pseudization of the nuclear cusp is of no relevant influence on HOMO-LUMO gaps or excitation energies. We have also shown that based on the same considerations that led to the construction of CAM-B3LYP, a new xc functional CAM-O3LYP can be constructed, which shows improved performance over CAM-B3LYP in systems where the latter fails, and yields comparable accuracy for systems where CAM-B3LYP typically fares well. This demonstrates that the flexibility of "à-la-carte" combinations of xc functionals can help in obtaining excitations of higher accuracy over a larger range of systems.

\section{ASSOCIATED CONTENT}

\section{S Supporting Information}

The Supporting Information is available free of charge on the ACS Publications website at DOI: 10.1021/acs.jctc.8b00069.
Convergence of the HOMO-LUMO gaps in water using MT pseudopotentials; simulation supercell dimensions for all systems; additional excitation energies for the molecules of ref 9 that have not been tabulated in the main text (PDF)

\section{AUTHOR INFORMATION}

\section{Corresponding Author}

*E-mail: ursula.roethlisberger@epfl.ch.

\section{ORCID}

Martin P. Bircher: 0000-0002-6905-3130

Ursula Rothlisberger: 0000-0002-1704-8591

\section{Notes}

The authors declare no competing financial interest.

\section{ACKNOWLEDGMENTS}

The authors thank J. Magnus Haugaard Olsen for providing a molecular dynamics snapshot of the retinal protonated Schiff base, Valery Weber for comments on the implementation of the new xc driver, and the CADMOS project for providing computing time on the BG/Q. U.R. gratefully acknowledges financial support from the Swiss National Science Foundation Grant No. 200020-146645 and the NCCR MUST.

\section{REFERENCES}

(1) Hohenberg, P.; Kohn, W. Inhomogeneous Electron Gas. Phys. Rev. 1964, 136, B864-B871.

(2) Kohn, W.; Sham, L. J. Self-Consistent Equations Including Exchange and Correlation Effects. Phys. Rev. 1965, 140, A1133A1138.

(3) Runge, E.; Gross, E. K. U. Density-Functional Theory for TimeDependent Systems. Phys. Rev. Lett. 1984, 52, 997-1000.

(4) Casida, M.; Ipatov, A.; Cordova, F. In Time-Dependent Density Functional Theory; Marques, M. A., Ullrich, C. A., Nogueira, F., Rubio, A., Burke, K., Gross, E. K. U., Eds.; Springer: Berlin/Heidelberg, 2006; pp 243-257.

(5) Tawada, Y.; Tsuneda, T.; Yanagisawa, S.; Yanai, T.; Hirao, K. A long-range-corrected time-dependent density functional theory. $J$. Chem. Phys. 2004, 120, 8425-8433.

(6) Langreth, D. C.; Perdew, J. P. Theory of nonuniform electronic systems. I. Analysis of the gradient approximation and a generalization that works. Phys. Rev. B: Condens. Matter Mater. Phys. 1980, 21, 54695493.

(7) Tozer, D. J.; Amos, R. D.; Handy, N. C.; Roos, B. O.; SerranoANDRES, L. Does density functional theory contribute to the understanding of excited states of unsaturated organic compounds? Mol. Phys. 1999, 97, 859-868.

(8) Becke, A. D. A new mixing of Hartree-Fock and local densityfunctional theories. J. Chem. Phys. 1993, 98, 1372-1377.

(9) Peach, M. J. G.; Benfield, P.; Helgaker, T.; Tozer, D. J. Excitation energies in density functional theory: An evaluation and a diagnostic test. J. Chem. Phys. 2008, 128, 044118.

(10) Iikura, H.; Tsuneda, T.; Yanai, T.; Hirao, K. A long-range correction scheme for generalized-gradient-approximation exchange functionals. J. Chem. Phys. 2001, 115, 3540-3544.

(11) Yanai, T.; Tew, D. P.; Handy, N. C. A new hybrid exchangecorrelation functional using the Coulomb-attenuating method (CAMB3LYP). Chem. Phys. Lett. 2004, 393, 51-57.

(12) Peverati, R.; Truhlar, D. G. Quest for a universal density functional: the accuracy of density functionals across a broad spectrum of databases in chemistry and physics. Philos. Trans. R. Soc., A 2014, 372, 20120476.

(13) Stephens, P. J.; Devlin, F. J.; Chabalowski, C. F.; Frisch, M. J. Ab Initio Calculation of Vibrational Absorption and Circular Dichroism 
Spectra Using Density Functional Force Fields. J. Phys. Chem. 1994, 98, 11623-11627.

(14) Perdew, J. P.; Burke, K.; Ernzerhof, M. Generalized Gradient Approximation Made Simple. Phys. Rev. Lett. 1996, 77, 3865-3868.

(15) Jensen, L.; Govind, N. Excited States of DNA Base Pairs Using Long-Range Corrected Time-Dependent Density Functional Theory. J. Phys. Chem. A 2009, 113, 9761-9765.

(16) Arulmozhiraja, S.; Coote, M. L. ${ }^{1} L_{a}$ and ${ }^{1} L_{b}$ States of Indole and Azaindole: Is Density Functional Theory Inadequate? J. Chem. Theory Comput. 2012, 8, 575-584.

(17) Valsson, O.; Campomanes, P.; Tavernelli, I.; Rothlisberger, U.; Filippi, C. Rhodopsin Absorption from First Principles: Bypassing Common Pitfalls. J. Chem. Theory Comput. 2013, 9, 2441-2454.

(18) Laktionov, A.; Rothlisberger, U., unpublished, 2014.

(19) Perdew, J. P.; Ernzerhof, M.; Burke, K. Rationale for mixing exact exchange with density functional approximations. J. Chem. Phys. 1996, 105, 9982-9985.

(20) Adamo, C.; Barone, V. Toward reliable density functional methods without adjustable parameters: The PBE0 model. J. Chem. Phys. 1999, 110, 6158-6170.

(21) Peach, M. J. G.; Cohen, A. J.; Tozer, D. J. Influence of Coulomb-attenuation on exchange-correlation functional quality. Phys. Chem. Chem. Phys. 2006, 8, 4543-4549.

(22) Rohrdanz, M. A.; Herbert, J. M. Simultaneous benchmarking of ground- and excited-state properties with long-range-corrected density functional theory. J. Chem. Phys. 2008, 129, 034107.

(23) Riffet, V.; Jacquemin, D.; Cauët, E.; Frison, G. Benchmarking dft and td-dft Functionals for the Ground and Excited States of Hydrogen-Rich Peptide Radicals. J. Chem. Theory Comput. 2014, 10, 3308-3318.

(24) Eriksen, J. J.; Sauer, S. P.; Mikkelsen, K. V.; Christiansen, O.; Jensen, H. J. A.; Kongsted, J. Failures of TDDFT in describing the lowest intramolecular charge-transfer excitation in para-nitroaniline. Mol. Phys. 2013, 111, 1235-1248.

(25) Sevilla, M. D. Comment on 'Excited States of DNA Base Pairs Using Long-Range Corrected Time-Dependent Density Functional Theory'. J. Phys. Chem. A 2009, 113, 11093-11094.

(26) Serrano-Andrés, L.; Fülscher, M. P. Theoretical Study of the Electronic Spectroscopy of Peptides. III. Charge-Transfer Transitions in Polypeptides. J. Am. Chem. Soc. 1998, 120, 10912-10920.

(27) Odelius, M.; Laikov, D.; Hutter, J. Excited state geometries within time-dependent and restricted open-shell density functional theories. J. Mol. Struct.: THEOCHEM 2003, 630, 163-175 (WATOC '02 Special Issue).

(28) Kronik, L.; Stein, T.; Refaely-Abramson, S.; Baer, R. Excitation Gaps of Finite-Sized Systems from Optimally Tuned Range-Separated Hybrid Functionals. J. Chem. Theory Comput. 2012, 8, 1515-1531.

(29) Heyd, J.; Scuseria, G. E.; Ernzerhof, M. Hybrid functionals based on a screened Coulomb potential. J. Chem. Phys. 2003, 118, 82078215

(30) CPMD, IBM Corp. and Max-Planck Institut Stuttgart, 20002018; www.cpmd.org.

(31) Weber, V.; Bekas, C.; Laino, T.; Curioni, A.; Bertsch, A.; Futral, S. Shedding Light on Lithium/Air Batteries Using Millions of Threads on the BG/Q Supercomputer. 2014 IEEE 28th International Parallel and Distributed Processing Symposium, 2014; pp 735-744.

(32) Gygi, F.; Baldereschi, A. Self-consistent Hartree-Fock and screened-exchange calculations in solids: Application to silicon. Phys. Rev. B: Condens. Matter Mater. Phys. 1986, 34, 4405-4408.

(33) Broqvist, P.; Alkauskas, A.; Pasquarello, A. Hybrid-functional calculations with plane-wave basis sets: Effect of singularity correction on total energies, energy eigenvalues, and defect energy levels. Phys. Rev. B: Condens. Matter Mater. Phys. 2009, 80, 085114.

(34) Xu, X.; Goddard, W. A. The X3LYP extended density functional for accurate descriptions of nonbond interactions, spin states, and thermochemical properties. Proc. Natl. Acad. Sci. U. S. A. 2004, 101, 2673-2677.
(35) Becke, A. D. Density-functional exchange-energy approximation with correct asymptotic behavior. Phys. Rev. A: At., Mol, Opt. Phys. 1988, 38, 3098-3100.

(36) Perdew, J. P. Electronic structure of solids; Akademie Verlag: Berlin, 1991; Vol. 11.

(37) Rostov, I. V.; Amos, R. D.; Kobayashi, R.; Scalmani, G.; Frisch, M. J. Studies of the Ground and Excited-State Surfaces of the Retinal Chromophore using CAM-B3LYP. J. Phys. Chem. B 2010, 114, 55475555.

(38) Sneskov, K.; Olsen, J. M. H.; Schwabe, T.; Hattig, C.; Christiansen, O.; Kongsted, J. Computational screening of one- and two-photon spectrally tuned channelrhodopsin mutants. Phys. Chem. Chem. Phys. 2013, 15, 7567-7576.

(39) Palczewska, G.; Vinberg, F.; Stremplewski, P.; Bircher, M. P.; Salom, D.; Komar, K.; Zhang, J.; Cascella, M.; Wojtkowski, M.; Kefalov, V. J.; Palczewski, K. Human infrared vision is triggered by two-photon chromophore isomerization. Proc. Natl. Acad. Sci. U. S. A. 2014, 111, E5445-E5454.

(40) Aidas, K.; Angeli, C.; Bak, K. L.; Bakken, V.; Bast, R.; Boman, L.; Christiansen, O.; Cimiraglia, R.; Coriani, S.; Dahle, P.; Dalskov, E. K.; Ekström, U.; Enevoldsen, T.; Eriksen, J. J.; Ettenhuber, P.; Fernández, B.; Ferrighi, L.; Fliegl, H.; Frediani, L.; Hald, K.; Halkier, A.; Hättig, C.; Heiberg, H.; Helgaker, T.; Hennum, A. C.; Hettema, H.; Hjertenæs, E.; Høst, S.; Høyvik, I.-M.; Iozzi, M. F.; Jansík, B.; Jensen, H. J. Aa.; Jonsson, D.; Jørgensen, P.; Kauczor, J.; Kirpekar, S.; Kjærgaard, T.; Klopper, W.; Knecht, S.; Kobayashi, R.; Koch, H.; Kongsted, J.; Krapp, A.; Kristensen, K.; Ligabue, A.; Lutnæs, O. B.; Melo, J. I.; Mikkelsen, K. V.; Myhre, R. H.; Neiss, C.; Nielsen, C. B.; Norman, P.; Olsen, J.; Olsen, J. M. H.; Osted, A.; Packer, M. J.; Pawlowski, F.; Pedersen, T. B.; Provasi, P. F.; Reine, S.; Rinkevicius, Z.; Ruden, T. A.; Ruud, K.; Rybkin, V. V.; Sałek, P.; Samson, C. C. M.; de Merás, A. S.; Saue, T.; Sauer, S. P. A.; Schimmelpfennig, B.; Sneskov, K.; Steindal, A. H.; Sylvester-Hvid, K. O.; Taylor, P. R.; Teale, A. M.; Tellgren, E. I.; Tew, D. P.; Thorvaldsen, A. J.; Thøgersen, L.; Vahtras, O.; Watson, M. A.; Wilson, D. J. D.; Ziolkowski, M.; Ågren, $\mathrm{H}$. The Dalton quantum chemistry program system. WIREs Comput. Mol. Sci. 2014, 4, 269-284.

(41) Frisch, M. J.; Trucks, G. W.; Schlegel, H. B.; Scuseria, G. E.; Robb, M. A.; Cheeseman, J. R.; Scalmani, G.; Barone, V.; Mennucci, B.; Petersson, G. A.; Nakatsuji, H.; Caricato, M.; Li, X.; Hratchian, H. P.; Izmaylov, A. F.; Bloino, J.; Zheng, G.; Sonnenberg, J. L.; Hada, M.; Ehara, M.; Toyota, K.; Fukuda, R.; Hasegawa, J.; Ishida, M.; Nakajima, T.; Honda, Y.; Kitao, O.; Nakai, H.; Vreven, T.; Montgomery, J. A., Jr.; Peralta, J. E.; Ogliaro, F.; Bearpark, M.; Heyd, J. J.; Brothers, E.; Kudin, K. N.; Staroverov, V. N.; Kobayashi, R.; Normand, J.; Raghavachari, K.; Rendell, A.; Burant, J. C.; Iyengar, S. S.; Tomasi, J.; Cossi, M.; Rega, N.; Millam, J. M.; Klene, M.; Knox, J. E.; Cross, J. B.; Bakken, V.; Adamo, C.; Jaramillo, J.; Gomperts, R.; Stratmann, R. E.; Yazyev, O.; Austin, A. J.; Cammi, R.; Pomelli, C.; Ochterski, J. W.; Martin, R. L.; Morokuma, K.; Zakrzewski, V. G.; Voth, G. A.; Salvador, P.; Dannenberg, J. J.; Dapprich, S.; Daniels, A. D.; Farkas, A.; Foresman, J. B.; Ortiz, J. V.; Cioslowski, J.; Fox, D. J. Gaussian09, Revision D.01; Gaussian Inc.: Wallingford, CT,2009.

(42) Dunning, T. H. Gaussian basis sets for use in correlated molecular calculations. I. The atoms boron through neon and hydrogen. J. Chem. Phys. 1989, 90, 1007-1023.

(43) Van Lenthe, E.; Baerends, E. J. Optimized Slater-type basis sets for the elements 1-118. J. Comput. Chem. 2003, 24, 1142-1156.

(44) te Velde, G.; Bickelhaupt, F. M.; Baerends, E. J.; Fonseca Guerra, C.; van Gisbergen, S. J. A.; Snijders, J. G.; Ziegler, T. Chemistry with ADF. J. Comput. Chem. 2001, 22, 931-967.

(45) Baerends, E. J.; Ziegler, T.; Atkins, A. J.; Autschbach, J.; Bashford, D.; Baseggio, O.; Bérces, A.; Bickelhaupt, F. M.; Bo, C.; Boerritger, P. M.; Cavallo, L.; Daul, C.; Chong, D. P.; Chulhai, D. V.; Deng, L.; Dickson, R. M.; Dieterich, J. M.; Ellis, D. E.; van Faassen, M.; Ghysels, A.; Giammona, A.; van Gisbergen, S. J. A.; Goez, A.; Götz, A. W.; Gusarov, S.; Harris, F. E.; van den Hoek, P.; Hu, Z.; Jacob, C. R.; Jacobsen, H.; Jensen, L.; Joubert, L.; Kaminski, J. W.; van Kessel, G.; König, C.; Kootstra, F.; Kovalenko, A.; Krykunov, M.; van Lenthe, E.; 
McCormack, D. A.; Michalak, A.; Mitoraj, M.; Morton, S. M.; Neugebauer, J.; Nicu, V. P.; Noodleman, L.; Osinga, V. P.; Patchkovskii, S.; Pavanello, M.; Peeples, C. A.; Philipsen, P. H. T.; Post, D.; Pye, C. C.; Ramanantoanina, H.; Ramos, P.; Ravenek, W.; Rodríguez, J. I.; Ros, P.; Rüger, R.; Schipper, P. R. T.; Schlüns, D.; van Schoot, H.; Schreckenbach, G.; Seldenthuis, J. S.; Seth, M.; Snijders, J. G.; Solà, M.; Stener, M.; Swart, M.; Swerhone, D.; te Velde, G.; Tognetti, V.; Vernooijs, P.; Versluis, L.; Visscher, L.; Visser, O.; Wang, F.; Wesolowski, T. A.; van Wezenbeek, E. M.; Wiesenekker, G.; Wolff, S. K.; Woo, T. K.; Yakovlev, A. L. ADF2017; SCM, Theoretical Chemistry, Vrije Universiteit: Amsterdam, The Netherlands, 2017; https://www.scm.com.

(46) Fonseca Guerra, C.; Snijders, J. G.; te Velde, G.; Baerends, E. J. Towards an order-N DFT method. Theor. Chem. Acc. 1998, 99, 391403.

(47) Hirata, S.; Head-Gordon, M. Time-dependent density functional theory within the Tamm-Dancoff approximation. Chem. Phys. Lett. 1999, 314, 291-299.

(48) Troullier, N.; Martins, J. L. Efficient pseudopotentials for planewave calculations. Phys. Rev. B: Condens. Matter Mater. Phys. 1991, 43, 1993-2006.

(49) Goedecker, S.; Teter, M.; Hutter, J. Separable dual-space Gaussian pseudopotentials. Phys. Rev. B: Condens. Matter Mater. Phys. 1996, 54, 1703-1710.

(50) Martyna, G. J.; Tuckerman, M. E. A reciprocal space based method for treating long range interactions in ab initio and force-fieldbased calculations in clusters. J. Chem. Phys. 1999, 110, 2810-2821.

(51) Bekas, C.; Curioni, A.; Andreoni, W. Atomic wavefunction initialization in ab initio molecular dynamics using distributed Lanczos. Parallel Computing 2008, 34, 441-450 (Parallel Matrix Algorithms and Applications).

(52) Bekas, C.; Curioni, A. Very large scale wavefunction orthogonalization in Density Functional Theory electronic structure calculations. Comput. Phys. Commun. 2010, 181, 1057-1068.

(53) Peach, M. J. G.; Tozer, D. J. Overcoming Low Orbital Overlap and Triplet Instability Problems in TDDFT. J. Phys. Chem. A 2012, 116, 9783-9789.

(54) Grimme, S.; Parac, M. Substantial Errors from Time-Dependent Density Functional Theory for the Calculation of Excited States of Large $\pi$ Systems. ChemPhysChem 2003, 4, 292-295.

(55) Bulliard, C.; Allan, M.; Wirtz, G.; Haselbach, E.; Zachariasse, K. A.; Detzer, N.; Grimme, S. Electron Energy Loss and DFT/SCI Study of the Singlet and Triplet Excited States of Aminobenzonitriles and Benzoquinuclidines: Role of the Amino Group Twist Angle. J. Phys. Chem. A 1999, 103, 7766-7772.

(56) Svartsov, Y. N.; Schmitt, M. Electronically excited states of water clusters of 7-azaindole: Structures, relative energies, and electronic nature of the excited states. J. Chem. Phys. 2008, 128, 214310.

(57) Kosenkov, D.; Slipchenko, L. V. Solvent Effects on the Electronic Transitions of p-Nitroaniline: A QM/EFP Study. J. Phys. Chem. A 2011, 115, 392-401.

(58) Handy, N. C.; Cohen, A. J. Left-right correlation energy. Mol. Phys. 2001, 99, 403-412.

(59) Lee, C.; Yang, W.; Parr, R. G. Development of the Colle-Salvetti correlation-energy formula into a functional of the electron density. Phys. Rev. B: Condens. Matter Mater. Phys. 1988, 37, 785-789.

(60) Hoe, W.-M.; Cohen, A. J.; Handy, N. C. Assessment of a new local exchange functional OPTX. Chem. Phys. Lett. 2001, 341, 319328.

(61) Baker, J.; Pulay, P. Assessment of the Handy-Cohen optimized exchange density functional for organic reactions. J. Chem. Phys. 2002, 117, 1441-1449.

(62) Baker, J.; Pulay, P. Assessment of the OLYP and O3LYP density functionals for first-row transition metals. J. Comput. Chem. 2003, 24, $1184-1191$.

(63) Xu, X.; Goddard, W. A. Assessment of Handy-Cohen Optimized Exchange Density Functional (OPTX). J. Phys. Chem. A 2004, 108, 8495-8504.
(64) Lange, A. W.; Herbert, J. M. Both Intra- and Interstrand ChargeTransfer Excited States in Aqueous B-DNA Are Present at Energies Comparable To, or Just Above, the $1 \pi \pi^{*}$ Excitonic Bright States. J. Am. Chem. Soc. 2009, 131, 3913-3922. 